\title{
Smart Grid System and Efficient Location Finder for Renewable Power Plant based on One Sun One World One Grid
}

\author{
Jyoti Mante Khurpade \\ Department of Computer Engg., \\ MIT-WPU \\ Pune, Maharashtra, India \\ jyoti.khurpade@mitwpu.edu.in \\ Aditya Pravin Chandsare \\ Department of Computer Engg., \\ MIT-WPU \\ Pune, Maharashtra, India \\ apchandsare@gmail.com
}

\author{
Vaibhav Kailas Gurap \\ Department of Computer Engg., \\ MIT-WPU \\ Pune, Maharashtra, India \\ gurapvaibhav@gmail.com \\ Omkar Ramdas Irole \\ Department of Computer Engg., \\ MIT-WPU \\ Pune, Maharashtra, India \\ omkarirole5556@gmail.com
}

\author{
Rudra Pramod Chopde \\ Department of Computer Engg., \\ MIT-WPU \\ Pune, Maharashtra, India \\ rudrachopde@gmail.com
}

\begin{abstract}
Fossil fuels are the main source of energy and also one of the main contributor for Global Warming. Use of these fossil fuels can be replaced by Clean energy sources such as Solar, Wind, Hydro, etc. With the view of increasing the use of Clean energy, a project named One Sun One World One Grid has been launched by the Government of India. The One Sun One World One Grid(OSOWOG) is based on a mantra "The Sun Never Sets for the whole World" hence we can generate round the clock electricity from the Sun. The one of the major problem in this OSOWOG project is switching between renewable and conventional sources of energy as it requires skill manpower at every main grid. In this paper, two methods are proposed by which such projects can be accomplished with less manpower and in a cost efficient way. The first system is an Smart Grid System and the second system is an Efficient location finder for Renewable Power Plants. These both systems will be build using Neural Networks.
\end{abstract}

Keywords-One Sun One World One Grid(OSOWOG), Machine Learning, Neural Networks.

\section{INTRODUCTION}

The idea of One Sun One World One Grid was first floated by Honourable Prime Minister Narendra Modi during the first assembly of International Solar Alliance also know as ISA. The vision behind OSOWOG is "The Sun Never Sets" and it is present at some location at any given point of time and thus power can be transmitted from the one region which will be the generation point to the another needed region which lacks in generation of solar energy at that point of time. We in this paper are proposing two systems which will help in completing this OSOWOG project and also not just limiting to Solar power but also considering other sources such as Wind and Hydro. The first system is an Smart Grid System and the second system is an Efficient location finder for Renewable Power Plants. These both systems will be build using Neural Networks. The Smart Grid System consists of four modules: Region Wise Hourly Power Consumption Prediction, Region Wise Hourly Power Generation, Classifying regions as Surplus or Deficit, Transmitting Surplus to Deficit. The Efficient Location Finder consists of three modules: Getting satellite image and
Classifying it as Barren land or not, Calculating the Efficiency of the Barren land for the wind and solar power generation.

\section{LITERATURE SURVEY}

Predicting Power Consumption is an difficult an complex task. Despite of the consumption being unpredictable the authors of this paper "The daily and hourly energy consumption and load forecasting using artificial neural network method: a case study using a set of 93 households in Portugal" [1] have described a method using Artificial Neural Network which can predict the hourly power consumption by using just the time stamp. The authors used Levenberg-Marquardt Algorithm which combines the robustness of the steepest-descent method with the quadratic convergence of the Gauss-Newton. The author used Hourly Boolean Metering System. The input layer consists of 11 neurons: 5 neurons for last 5 hours load values, 1 neuron for load 24 hours ago and 5 neurons for binary encoding forecast time. The authors achieved a good accuracy with the $\mathrm{R}^{2}$ value as $98.5 \%$.

For predicting region wise power generation we are only focusing on Renewable Energy Sources. The authors of the paper [2] have proposed an Ensemble Model for predicting Solar Power Generation. The proposed model is divided in four stages: Improving Computational Difficulty and avoiding the difficulty of selecting weather data which is done using Cluster analysis, Then the random forests with different parameters is established for different weather regimes, ridge regression is used to obtain weights. The final forecast is calculated using the weights. The authors achieved Mean Absolute Error of 3.90\%. The authors of the paper [3] have proposed NARX(Non-linear auto-regressive with exogenous input). The proposed model has connections form each neuron in hidden layer to every neurons in the output layer. The Mean Absolute Error of the proposed model is about 0.9 .

Satellite Image Classification is a part of computer vision which is in boom and being researched from past few decades. To find barren lands we need a Model which can 
classify a land as barren or not. The paper [4] proposes 1D CNN Classifier with 8 hidden layers which include 5 Conv layers and 3 Max Pooling layers. The output layer has a softmax activation function. The author have achieved an accuracy of approximately $89.18 \%$.

The paper [5] proposes a formula to estimate the yearly solar power generation potential.

$\mathrm{GP}=\mathrm{SR} \times \mathrm{CA} \times \mathrm{AF} \times \eta$

Eq.(1) Yearly Solar Power Generation Potential

Where, GP stands for Annual power generation potential in $\mathrm{kWh} /$ day, SR stands for Annual solar radiation received per unit horizontal area in $\mathrm{kWh} / \mathrm{m}^{2} /$ day, CA stands for the available area in $\mathrm{m}^{2}$, AF stands for area factor which is $70 \%$ and $\eta$ stands for PV panel's efficiency.

\section{Methodology For SMART GRID SYSTEM}

This section of the paper discusses the methodology used for implementing Smart Grid System. As discussed before this system consists of 4 modules : Region Wise Hourly Power Consumption Prediction, Region Wise Hourly Power Generation, Classifying regions as Surplus or Deficit, Transmitting Surplus to Deficit.

\section{A. Power Consumption Prediction}

For predicting power consumption we are using PJM dataset available at kaggle. The dataset contains hourly load values from 2014 to 2018 . For prediction we can use KNeighborsRegressor which yields and accuracy of $92 \%$ on test sets. But using the architecture discussed in [1] we can achieve accuracy of $97 \%$.

\section{B. Power Generation Prediction}

We need to prediction power generation only for renewable source as these generation of these sources depend on various factors such as weather,etc. where as the generation of conventional sources is fixed and doesn't change due to any external changes. We would be focusing on solar and wind energies for this work. Solar energy depends on several factors such as time, season, topography of the region, clearness of the sky. Using all these factors as inputs for the architecture discussed in [2] we can predict hourly solar power generation using Neural Networks.

The wind power generation also depend on various factors but the three main factors are wind speed, air density and blade radius. Using the same architecture used for solar energy generation we can predict hourly wind power generation.

\section{Classifying regions}

After predicting power the generation and consumption the next task finding areas which can not fulfill its power requirement(through renewable sources) and the areas which can fulfill their power requirement and can also share it's surplus generation with other areas which need energy. The table below describes how the areas would be classified. These classified will be done on hourly basis using Classification algorithm and decision tree to distinguish between DeficitR and Deficit.
TABLE I. CLASSIFICATION OF REGIONS

\begin{tabular}{|c|c|}
\hline Classes & Description \\
\hline Surplus & The area has Surplus Energy Generation \\
\hline DeficitR & $\begin{array}{c}\text { The area has Deficit Energy Generation but the } \\
\text { requirement will be fulfilled by some other area which } \\
\text { has Surplus Generation }\end{array}$ \\
\hline Balanced & The area has balanced energy generation \\
\hline Deficit & $\begin{array}{c}\text { The area has Deficit power generation but can use its } \\
\text { conventional energy sources }\end{array}$ \\
\hline Worst & $\begin{array}{c}\text { The area has Deficit power generation and has no source } \\
\text { which can fulfill its demand }\end{array}$ \\
\hline
\end{tabular}

\section{Transmitting}

The transmission and classification task goes hand in hand. This will be the final step of this system. In the transmission step if an area is going to receive energy from another area, the transmitting(Surplus) area acts as master node for the receiving(DeficitR) area. To determine which area to act as transmitting and receiving, we will be using Artificial Neural Network which take various factors in consideration such as transmission loss, priority of the area,etc . For Deficit areas the conventional source of energy will act as master node.

\section{Methodology For EFFicient LocAtion FindeR}

This section of the paper discusses the methodology used for implementing Efficient Location Finder. This system consists of 3 modules : Getting satellite image and Classifying it as Barren land or not, Calculating the Efficiency of the Barren land for the wind and solar power generation.

\section{A. Getting Satellite Image}

The task of getting satellite image is done using the WebDriver from the Selenium package. The WebDriver is an open source API which is used to automate web application. It is mainly used for testing purpose but here we will be using it to get satellite image. Before a getting satellite image we need to get some random coordinates. A coordinate is a combination of latitude and longitude which uniquely identifies a particular location. To generate random coordinate we need to first decide that do we want to generate random coordinate throughout the world or within a specific region. To generate random coordinate through the world we use the python uniform function from the random package as shown below:-

\section{from random import uniform}

$$
\text { lat, lon = uniform }(-180,180) \text {, uniform }(-90,90)
$$

To generate random coordinate for a specific region we need to find points i.e. latitude of the eastern most corner, latitude of the western most corner, longitude of the northern most corner, longitude of the southern most corner. Then we can use the uniform function to generate coordinate like:-

from random import uniform

$$
\begin{aligned}
& \text { lat }=\text { uniform(eastLat,westLat }) \\
& \text { lon = uniform(northLon,southLon) }
\end{aligned}
$$


Eg. Generating random coordinates in India

lat $=$ random.uniform $(8.0883,34.1526)$

lon $=$ random.uniform $(68.7692,87.046191)$

The second task after generating random coordinate is to check whether it is land or water, as there is a $70 \%$ of chance of getting a coordinate in ocean as it covers $70 \%$ of our earths area. As per ourworlddata.org $19 \%$ of the land cover is barren land hence finding a barren takes time.

After classifying the coordinate the third task is to get satellite image of that particular coordinate from Google Earth using selenium WebDriver. The satellite image captured by selenium after zooming in 3 times is approximately $8.963 \mathrm{~km}^{2}$. After capturing the satellite image the next task is to classify is as barren or not.

\section{B. Classifying as barren land}

The satellite image captured by selenium is classified using Convolutional Neural Network. The dataset used for training is DeepSat(SAT-4) Airborne Dataset with 4 lakh training images and 1 lakh testing images. The architecture of the CNN used is:-

TABLE II. CNN ARCHITECTURE

\begin{tabular}{|c|l|l|l|}
\hline Layer & \multicolumn{1}{|c|}{ Size } & No of Neurons & \multicolumn{1}{|c|}{$\begin{array}{c}\text { Activation } \\
\text { Function }\end{array}$} \\
\hline Conv2D & $3 \times 3$ & 16 & relu \\
\hline Conv2D & $3 \times 3$ & 32 & relu \\
\hline MaxPool2D & $2 \times 2$ & - & - \\
\hline Dropout & 0.5 & - & - \\
\hline Conv2D & $3 \times 3$ & 32 & relu \\
\hline Conv2D & $3 \times 3$ & 64 & relu \\
\hline MaxPool2D & $2 \times 2$ & - & - \\
\hline Dropout & 0.5 & - & - \\
\hline Flatten & - & - & relu \\
\hline Dense & - & 128 & - \\
\hline Dropout & 0.5 & - & softmax \\
\hline Dense & - & 4 & \\
\hline
\end{tabular}

The loss function used is 'categorical_crossentropy' with 'adam' optimizer. The accuracy on the test set achieved after 6 echos is $98.65 \%$.

\section{Calculating the Efficiency of the Barren Land}

a) For solar power plant: As discussed in the [5] paper we can calculate General Potential of location using the following formula:-

$\mathrm{GP}=\mathrm{SR} \times \mathrm{CA} \times \mathrm{AF} \times \eta$

Where, GP stands for Annual power generation potential in $\mathrm{kWh}$ /day, SR stands for Annual solar radiation received per unit horizontal area in $\mathrm{kWh} / \mathrm{m}^{2} /$ day, CA stands for the available area in $\mathrm{m}^{2}$, AF stands for area factor which is $70 \%$ and $\eta$ stands for PV panel's efficiency

\section{CONCLUSION}

The Smart Grid System which is working on Neural Network will help in taking critical decisions for electricity transmission and deciding the power source. The Efficient Location Finder will help in dealing the hectic job of finding efficient location through out the world which can only be done by skilled engineers. Hence, reducing the need of skilled people at every spot. The integration of these both systems will help a lot in completing the One Sun One World One Grid and also as it works using Neural Networks the systems will automatically adapt from the newly generated data. Thus making the system compatible with any future changes.

\section{REFERENCES}

[1] Fillipe Rodrigues, Carlos Carderia, and J. M. F. Calado, "The daily and hourly energy consumption and load forecasting using artificial neural network method: a case study using a set of 93 households in Portugal," The Mediterranean Green Energy Forum 2014, MGEF-14, December 2014.

[2] Cheng Pan, Jie Tan, "Day-Ahead Hourly Forecasting of Solar Generation Based on Cluster Analysis and Ensemble Model", IEEExplore, August 2019

[3] Ankita Singh, Gurtej K., Gourav Jain, Faraz Nayyar, MM Tripathi, "Short Term Wind Speed and Power Forecasting in Indian and UK Wind Power Frams",

[4] Yang Song, Zhifie Zhang, Razieh Kaviani Baghbaderani, Fanqi Wang, Ying Qu, Craig Stuttsy, Hairong Qi, "Land Cover Classification for Satellite Images through 1D CNN," $201910^{\text {th }}$ Workshop on Hyperspectral Imaging and Signal Processing: Evolution in Remote Sensing (WHISPERS).

[5] Ghazanfar Khan, Shikha Rathi,"Optimal Site Selection for Solar PV Power Plant in an Indian State Using Geographical Information System (GIS)", International Journal of Emerging Engineering Research and Technology, October 2014 